\title{
Epidemiological Factors Associated With Caligus rogercresseyi Infection, Abundance, and Spatial Distribution in Southern Chile
}

\section{OPEN ACCESS}

Edited by:

Jorge G. Farias,

University of La Frontera, Chile

Reviewed by:

Sandra Bravo,

Austral University of Chile, Chile

Raphael Victor Vanderstichel,

Long Island University, United States

Patricio Ulloa-Rodríguez,

Catholic University of Maule, Chile John Quiñones Diaz,

University of La Frontera, Chile

${ }^{*}$ Correspondence: Fernando O. Mardones

femardones@uc.cl

Specialty section: This article was submitted to Veterinary Epidemiology and Economics,

a section of the journal

Frontiers in Veterinary Science

Received: 14 August 2020

Accepted: 07 July 2021

Published: 20 August 2021

Citation:

Lepe-López M, Escobar-Dodero J,

Rubio D, Alvarez J, Zimin-Veselkoff N and Mardones FO (2021)

Epidemiological Factors Associated With Caligus rogercresseyi Infection, Abundance, and Spatial Distribution in Southern Chile.

Front. Vet. Sci. 8:595024. doi: 10.3389/fvets.2021.595024

\begin{abstract}
Manuel Lepe-López ${ }^{1,2}$, Joaquín Escobar-Dodero ${ }^{3}$, Daniel Rubio ${ }^{4}$, Julio Alvarez ${ }^{5,6}$, Natalia Zimin-Veselkoff ${ }^{4,7}$ and Fernando O. Mardones ${ }^{4,7 *}$

1 PhD Program in Conservation Medicine, Facultad de Ciencias de la Vida, Universidad Andres Bello, Santiago, Chile, ${ }^{2}$ Facultad de Ciencias de la Vida, Centro de Investigación para la Sustentabilidad, Universidad Andres Bello, Santiago, Chile, ${ }^{3}$ Department of Veterinary Population Medicine, University of Minnesota, St. Paul, MN, United States, ${ }^{4}$ EPIVET Analysis \& Solutions, Santiago, Chile, ${ }^{5}$ Centro de Vigilancia Sanitaria Veterinaria NISAVET), Universidad Complutense, Madrid, Spain, ${ }^{6}$ Departamento de Sanidad Animal, Facultad de Veterinaria, Universidad Complutense, Madrid, Spain, ${ }^{7}$ Escuela de Medicina Veterinaria, Facultad de Agronomía e Ingeniería Forestal, Facultad de Ciencias Biológicas, Facultad de Medicina, Pontificia Universidad Católica de Chile, Santiago, Chile
\end{abstract}

Sea lice (Caligus rogercresseyi) are external parasites that affect farmed salmonids in Chile, and the scale of their sanitary and economic impact cannot be overstated. Even though space-time patterns suppose parasite aggregation, specific locations related to different infestation levels, as well as their associated factors across the geographic range involved, had not been investigated as of the writing of the present article. The understanding of the effects and factors entailed by the presence of $C$. rogercresseyi may be deemed a key element of Integrated Pest Management (IPM). In the present study, the multivariate spatial scan statistic was used to identify geographic areas and times of $C$. rogercresseyi infestation and to estimate the factors associated with such patterns. We used official C. rogercresseyi monitoring data at the farm level, with a set of 13 covariates, to provide adjustment within the analyses. The analyses were carried out for a period of 5 years (2012-2016), and they included three fish species (Salmo salar, Oncorhynchus mykiss, and Oncorhynchus kisutch) in order to assess the consistency of the identified clusters. A retrospective multinomial, spatial, and temporal scan test was implemented to identify farm clusters of either of the different categories of $C$. rogercresseyi infested farms: baseline, medium, and high, based on the control chemical threshold established by the health authority. The baseline represents adequate farm performance against $C$. rogercresseyi infestation. Then, production and environmental factors of the medium and high infestation farms were compared with the baseline using regression techniques. The results revealed a total of 26 clusters ( $p<0.001$ ), of which 12 correspond to baseline, 1 to medium, and the remaining 13 to high infestation clusters. In general, baseline clusters are detected in a latitudinal gradient on estuarine areas, with increasing relative risks to complex island water systems. There is a spatial structure in specific sites, north of Los Lagos Region and central Aysén Region, with high infestation clusters and epidemic peaks during 2013. In addition, average weight, salmon species, chemotherapeutants, latitude, temperature, salinity, and year category are factors associated with these C. rogercresseyi 
patterns. Recommendations for an IPM plan are provided, along with a discussion that considers the involvement of stock density thresholds by salmon species and the spatial structure of the efficacy of chemical control, both intended to avoid the advance of resistance and to minimize environmental residues.

Keywords: sea lice, Caligus rogercresseyi, salmon farming, host-density, integrated pest management

\section{INTRODUCTION}

In the Southern Hemisphere, Caligus rogercresseyi is the external parasite affecting most farmed salmonid species (Salmo salar, Oncorhynchus mykiss, and Oncorhynchus kisutch), with enormous sanitary and economic costs. In Chile, the second largest producer worldwide and the largest producer of farmed salmonids in South America, the cost unit of Atlantic salmon (S. salar) increases by an average price of US $\$ 1.4 / \mathrm{kg}$, due to control measures against $C$. rogercresseyi (1). As a result of Chilean industry production, 953,296 tons/year at the end of 2019 (where S. salar represented 75\%, O. mykiss 9\%, and O. kisutch $16 \%$ of the total), the cost of these measures amounted to $\sim$ US\$ 1 billion (2). The life cycle of this copepod presents eight developmental stages. Three are planktonic, and the remaining five are parasitic (3). The non-parasitic stages include two naupliar stages and one copepodid or parasitic stage. The parasitic stages include four chalimus stages attached by the frontal filament to the fish, mounting male and female adults. The chromosome-scale whole genome sequence has been reported (4). Like other parasitic copepods of the Caligidae family (commonly named as sea lice), C. rogercresseyi feed on the mucus and tissue of the host, impairing both feed conversion and carcass quality, thus affecting the commercial value of the product (5). Atlantic salmon with C. rogercresseyi present skin abrasions, followed by osmotic problems, physiological stress, hematological alterations, reduced appetite, weight loss due to energy demand, and secondary bacterial or viral infestations (6-8).

In 2007, the Chilean National Fisheries Service (Sernapesca), acting in its role as a point of reference for the technical surveillance of the Salmon Technical Institute (INTESAL), implemented a national surveillance program for the monitoring of the abundant presence of $C$. rogercresseyi (9). This monitoring consists in taking random samples of 10 fish from four cages in each salmon farm, counting the parasitic stages and dividing those counts by the number of fish. From this sample, trained personnel carry out counts of chalimus, including male and non-gravid female adults, and adult gravid female stages of $C$. rogercresseyi. A cross-sectional retrospective analysis of 2007 data allowed to establish that C. rogercresseyi is widely distributed in Region X [Los Lagos] and Region XI [Aysén], with a decreasing epidemiological case occurrence in farms located in southern areas (10). Field studies suggest the influence of $C$. rogercresseyi infestation, based on the immune response of the fish host $(S$. salar and O. mykiss are most susceptible than O. kisutch), water temperature (biological cycle develops between 4 and $17^{\circ} \mathrm{C}$ ), water salinity (survival decays $<25 \mathrm{ppt}$ ), fish weight (host density), juvenile parasite count (the mucus covering on the fish protect these stages), and the number of chemical control events $(3,11,12)$. Subsequently, two analyses of risk factors, based on data from 2006 to 2007, displayed parasitic persistence (sea lice counts $>11$ ) associated with stocking density (over $22 \mathrm{~kg} \mathrm{~m}^{-3}$ ), the season of the year (increasing in autumn), depth of water at the farm and cage depth location $(>50 \mathrm{~m})$, geographical zones (there is a link between south areas and lower infestation), and chemical control performed 1 month before sampling $(13,14)$. Recently, a study using 7 years of Sernapesca regulatory data (between 2011 and 2017) suggests that the initial weight of smolt at seawater stocking (>156 g), cumulative degree-days $(5,547$ degree-days for Atlantic salmon, 3,411 degree-days for rainbow trout, and 2,630 degree-days for coho salmon), high fish density (odds ratio of 0.85 ), fish species (odds ratio of 1.50 for rainbow trout compared to Atlantic salmon), region (odds ratio of 0.65 for Region XI compared to Region X), and longitude (lower abundance at easterly locations) are associated factors with sea lice abundance (15).

After the sanitary crisis of infectious salmon anemia (virus ISA, reported by Sernapesca) in 2007 and new sanitary regulations enacted in 2012, salmon farming in Chile has been in constant expansion, including more than $\sim 600$ farms and adding Region XII [Magallanes] as a new geographical area (16, 17). To synchronize operating and rest activities, geographical regions X [Los Lagos], XI [Aysén], and XII [Magallanes] have been divided into $\sim 60$ epidemiological neighborhoods across different production and environmental conditions (2). The farms are active at dissimilar times, stocking different species of salmonids (S. salar, O. mykiss, and O. kisutch) and rotating active ingredients for sea lice chemical control. According to the dynamics of infectious diseases (18), C. rogercresseyi patterns are heterogeneous in space and time. A recent study, limited to three epidemiological neighborhoods with a persistent history of C. rogercresseyi infestation on northern Region $\mathrm{X}$ [Los Lagos] during 2012-2015, suggests temporal patterns with high peaks in one specific site exclusively during 2013 (19). Space-time patterns suppose parasitic aggregation across the geographic area occupied by salmon farming, with an increase in risk around predetermined sites. Also, the genetic analysis of $C$. rogercresseyi along a latitudinal range $\left(40^{\circ}-52^{\circ} \mathrm{S}\right)$ in southern Chile proposes genetic homogeneity, morphometric variability among sites, and longest parasites from $>52^{\circ} \mathrm{S}(20)$. However, location areas associated with different infestation levels of $C$. rogercresseyi throughout three regions of Chile had not been investigated yet.

The effort to reduce C. rogercresseyi infestation on farmed salmonids in Chile is centered on the use of chemical control, either in baths or in feed. Historically, from 1999 to 2007, 
emamectin benzoate was used exclusively in the control of sea lice, resulting in loss of sensitivity to this chemical (21). Later, peroxide hydroxide and deltamethrin were approved for use in 2007. Approval was also granted for the use of diflubenzuron in 2009 , cypermethrin in 2010, azamethiphos in 2013, lufenuron in 2017, and hexaflumuron in 2019 (22). Several investigations in loss of sensitivity, resistance, and chemical control failure suggest that frequently repeated exposure to these ingredients could change C. rogercresseyi molecular mechanisms of detoxification, transport the drugs along the biological membrane, and limit the ability to penetrate the copepod cuticle (21, 23-29). Biological control methods are being explored in Chile, with no significant effect either on the fecundity or survival of C. rogercresseyi (30).

In Chile, the dynamics of $C$. rogercresseyi maintain persistent levels of occurrence, which are associated with production practices shared by different farms and companies (fish stock densities). In the marine environment, different groups of farms cultivate a large number of susceptible fish hosts at the same time, becoming a source of infestation to neighboring farms and to themselves in a restricted spatial area (31). The spatial structure of $C$. rogercresseyi dynamics has been approached exclusively to evaluate the effect of chemical control, identifying a distance-dependent cluster response mediated by unknown factors $(24,25,29)$. In addition, in most salmon-producing regions, local authorities encourage the adoption of Integrated Pest Management (IPM) and the exploration and use of other non-chemical measures to reduce sea lice occurrence levels $(32,33)$. The exploration of the spatial and temporal patterns of $C$. rogercresseyi could reduce potential interactions with areas susceptible to infestation, serving as an IPM approach in the marine environment of Chile. Recently, during 2017, a C. rogercresseyi outbreak was identified in the Magallanes Region (south $49^{\circ} 16^{\prime} \mathrm{S}$ ), an area that includes only $10 \%$ of Chilean salmon production, and where supposedly the water temperature and salinity conditions are not ideal to the parasite life cycle $(10,13)$. If the IPM objective is to isolate susceptible farms from infested farms, then the problem of management centers on minimizing interaction in space and/or time within C. rogercresseyi sites of infestation. On the other hand, if the IPM objectives are to include the role of water currents and to reduce the transport of the planktonic stages, then the management of the problem must take into account the influence of this factor in different locations and/or times across the study area over the annual rainfall trend.

Effective IPM plans must be adapted to suit the regionally varying environmental and biological factors that affect the intensity of parasitic infestations. For example, the increase of superficial water temperature can accelerate the maturation of C. rogercresseyi eggs, taking 45 days at $10.3^{\circ} \mathrm{C}$, and only 18 days at $16.7^{\circ} \mathrm{C}$ in experimental conditions (3). Besides, copepodid survival time is 30 and $44 \%$ longer than at 15 and $20^{\circ} \mathrm{C} \mathrm{(34).} \mathrm{Low}$ salinity concentration due to freshwater discharge from rivers reduces the survival rate of parasitic stages (35). In addition, the time and magnitude of river discharge vary during the seasons, depending on rainfall patterns and the influence of salinity concentrations. The understanding of risk factors such as temperature and salinity can be another tool in the development of an IPM plan for sea lice in southern Chile.
The space-time occurrence of a disease can be addressed using cluster analysis techniques. Clustering is a term used to describe the aggregation of disease, in both specific locations and time windows, to understand different events of interest (36). In this context, we are using local clustering methods for the detection of the locations and times of different $C$. rogercresseyi levels in order to understand the factors that could influence these patterns. We are interested in the study of three C. rogercresseyi levels of infestation, based on the parasitic threshold proposed by the health authority in Chile (if a farm reports $C$. rogercresseyi gravid female counts $>3$, it is mandatory to apply chemical control to reduce loads). Therefore, we propose a multinomial modeling process. This includes infestation levels below the threshold, around the threshold, and infestation levels above the sanitary threshold to explore clustering patterns. The identification of spacetime clustering of different infestation levels of $C$. rogercresseyi allows us to make comparisons that could inform the targeted investigation of the factor associated, as well as support the understanding of how to achieve better management concerning C. rogercresseyi. The objectives of this study were to identify geographic areas and times of $C$. rogercresseyi infestation levels throughout every salmon farming area in Chile by space-time cluster outcome and to estimate the factors associated with such patterns.

\section{MATERIALS AND METHODS}

\section{Study Area}

Information about the abundance of $C$. rogercresseyi comes from salmon farms located along three geographical regions: Region X [Los Lagos], Region XI [Aysén], and Region XII [Magallanes]. This area in southern Chile is a complex system of fjords and islands with a latitudinal gradient that begins in Puerto Montt $(-41.4740,-72.9441)$ and ends around Dawson Island $(-54.0370,-70.7149)$ (Figure 1). The salmon farms are operated by large and medium-sized companies. They are located $3 \mathrm{~km}$ from other farms and at $1-2 \mathrm{~km}$ from the coast. Salmon farms are licensed-organized within space estates called epidemiological neighborhoods, defined as space polygons that share similar production and environmental conditions for each group of farms. The salmonid production cycle begins with the transfer of broodstock in autumn for extraction, fertilization, incubation, and maintenance of eggs and fingerlings $<10^{\circ} \mathrm{C}$. Fish will be subjected to light and temperature regimens to induce early smoltification come spring. Smolted fish (weight $>100 \mathrm{~g}$ ) are transferred to sea sites for a growing period in floating cages of up to 1 or 2 years for the harvest. Farms are monitored so that they enter either a 3-month rest period when harvesting or a 3-week health-check.

\section{Data}

The study was developed using MS Excel data from the Specific Sanitary Program for the Surveillance and Control of Caligidosis in Chile SSPSC (SalmonChile INTESAL, Santiago, Chile), which carries out weekly surveys from January 2012 to December 2016 (5 years) of C. rogercresseyi abundance data among different life stages, divided into three categories, namely, juveniles (Chalimus 


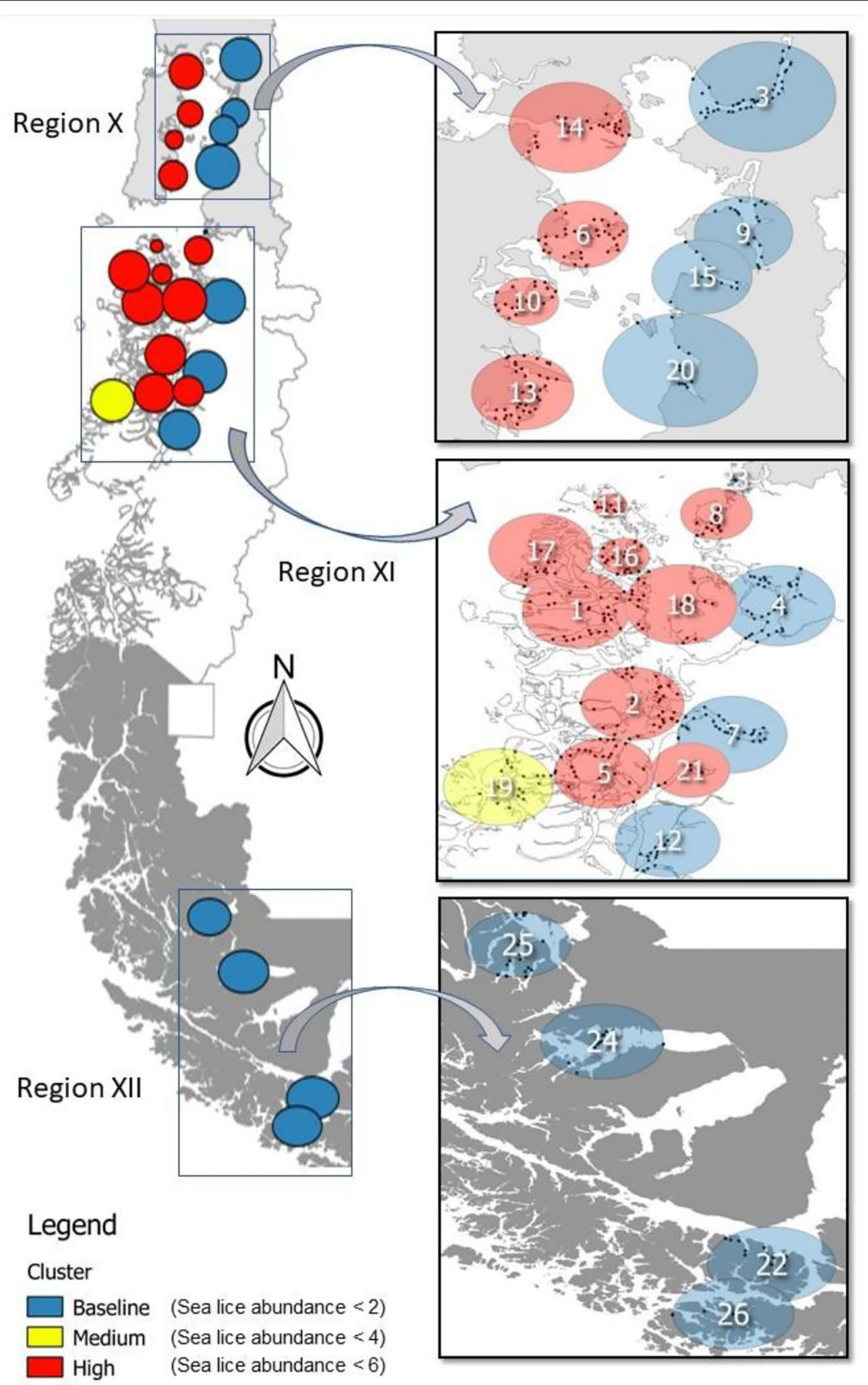

FIGURE 1 | Production area occupied by salmon farming Chile (left) covers three geographic regions during 2012-2016: Region X [Los Lagos], Region XI [Aysén], and Region XII [Magallanes]. Clusters resulting from a multinomial space-time SaTScan model are identified by the C. rogercresseyi infestation category colors (baseline-blue, medium-yellow, and high-red) and by number. Specific information for each cluster is provided in Table $\mathbf{1}$. 
I, II, III, and IV), mobile adults (males and no gravid females), and gravid female (egg sacs) (37). Sampling protocols at the farm level include the collection of 10 fish at random from four cages to estimate $C$. rogercresseyi abundance, date sample, year, month, farm ID, farm geographic coordinates (latitude and longitude), Chilean region, epidemiological neighborhood ID, and fish species (S. salar, O. mykiss, and O. kisutch). The database also presents weekly measurements of biomass (tons), number of fish (thousands), average weight $(\mathrm{kg})$, average water salinity (parts per thousand), average water temperature $\left({ }^{\circ} \mathrm{C}\right)$, chemical control counts, and chemotherapeutants (azamethiphos, emamectin benzoate, cypermethrin, deltamethrin, diflubenzuron, and none).

\section{Definition of C. rogercresseyi Infestation Levels and Data Management}

According to sanitary regulations in Chile, all farms must apply chemical control to avoid the C. rogercresseyi threshold (gravid female abundance $>3$ ). The gravid female stage is a reliable measurement due to the ease of identification with the naked eye in the field. Compared to other stages and repeated reports of this proxy, it can lead to an early harvest of farms (38). However, several $C$. rogercresseyi studies reported farms with abundances below the chemical control threshold (counts of 1 or 2), infested farms (counts around to the threshold [ 3]), and severely infested farms (values above the threshold) $(10,11,13$, 39). Based on this information, we categorize the data of each into three different infestation levels: baseline (farms with abundance $0-2$ ), medium (farms with abundance 2-4), and high (farms with abundance $>4$ ). The rationale behind this is to investigate the infested farms that never reached the parasitic threshold (baseline category), comparing them with those in the two higher levels of infestation observed (farms that reach the sanitary threshold and farms well above the threshold). Duplicate, aberrant, and writing errors were reviewed in order to clean the data. In addition, a weekly record of production and environmental factors was included by farm ID according to SSPSC data.

\section{Descriptive Analysis}

The MS Excel data were imported into the R software (version 4.0.2) for analysis (40). The definition of the level of infestation of a farm (prevalence and growth rate) was estimated as a previous guide to the temporal window choice of space-time analysis (plotting the temporal pattern of occurrence). The dataset was arranged according to C. rogercresseyi threshold abundance $>3$ (positive farm or case definition). Besides, it was used to describe a period prevalence, without distinction, between old and new cases by geographical region, using the "prevalence" package to estimate confidence intervals for proportions (weekly number of active farms as abundance $>3$ by study period/weekly number of active farms by study period), applying a 95\% confidence level through the Wald test. Also, we explore growth rate as a measure of disease spread over time (weekly number of new farms as abundance $>3$ by study period/the sum of the weeks where farms were active in the study period as a time of risk), using the "incidence" package (41) to estimate the growth rate. This package uses a simple log-linear regression model where the growth rate $(r)$ is the slope of the regression form $\log (y)=r \times t+$ $b$. Due to the observation of a peak in new cases from February to August 2013, we split the data to generate the growth rate before and after the peak.

\section{Space-Time Cluster Analysis}

A retrospective multinomial, spatial, and temporal scan test was implemented to identify farm clusters of either category of C. rogercresseyi infested farms: baseline (abundance 0-2), medium (abundance 2-4), and high (abundance $>4$ ). According to the baseline category and their statistical significance, clusters were prioritized for optimal C. rogercresseyi management. In the multinomial scan test, each observation is a case, and each case belongs to one of the three categories, evaluating whether there are any clusters where the distribution of cases is different from the rest of the study region as follows: let Cik be the number of observations in $C$. rogercresseyi abundance status $k$ for each farm $i$ (42). The probability of being in category $k$ was calculated for each farm, and likely circular-shape clusters were identified using a likelihood ratio test. The likelihood function for the multinomial model is written as

$$
L(Z, p 1, \ldots, p k, q 1, \ldots, q k) \propto \prod_{k-1}^{K}\left(\prod_{i \in Z} p k^{C i k} \prod_{i \notin Z} q k^{C i k}\right),
$$

where $p k$ and $q k$ were the probability of being in category $k$ within and outside window $Z$, respectively. To evaluate the statistical significance of the most likely cluster, Monte Carlo simulations $(n=999)$ were run. A previous analysis of sources of sea lice parasitic stages for salmon farms in Chile suggests that the exposure potential from neighboring farms is within a $30-\mathrm{km}$ radius (43). Therefore, the spatial analysis window was limited to $30 \mathrm{~km}$ of seaway distance, whereas the temporal window was 6 months. The space-time analysis was performed using SaTScan TM free license version 9.6 (www.satscan.org). Maps were constructed using open source QGIS Geographic Information System version 2.18 (www.qgis.org).

\section{Multinomial Logistic Model for Associated Factors}

Using a multinomial logistic regression model, production and environmental factors of the farms located within each infestation category of $C$. rogercresseyi (medium and high) were compared with those of farms located within the baseline category. This type of model is an extension of generalized linear models, allowing for an estimation of an unordered categorical response. The multinomial probability distribution is an extension of the binomial distribution. However, the multinomial logistic model tests the probability of being in a given category compared to other categories, where the relationships are thought of as relative risk ratios (RRR) (44). Production factors used as predictors were time (5 years: 20122016), average weight (kg), biomass (tons), number of fish (integer value), epidemiological neighborhood (administrative farms group), company (nominal name), species of fish (Atlantic salmon, rainbow trout, and coho salmon), number of chemical treatments (integer counts), and chemical treatment ingredient (azamethiphos, emamectin benzoate, cypermethrin, 
deltamethrin, and diflubenzuron). Environmental factors used as predictors were temperature (degrees centigrade), salinity (part per thousand), farm locality (longitude and latitude in decimal degrees), and geographical region of Chile (X, XI, and XII).

Collinearity among continuous predictors (time, average weight, biomass, number of fish, and number of chemical treatments) was assessed with pairwise scatterplot and Pearson correlation coefficients (45). We imported the MS Excel data into R statistical software v.3.6.0 (R Development Core Team) for the selection and validation of the model and then retained statistically significant factors in the final model. The multinomial model tests the probability or risk (thought of as $\mathrm{RRR}$ ) of being in a given category or level compared to other categories as follows:

$$
\ln \frac{p(Y=k)}{p(Y=\text { baseline category })}=\beta_{0}^{k}+\beta_{j}^{k} X_{j},
$$

where the coefficients $\beta_{0}^{k}+\beta_{j}^{k}$ of the $K-1$ category were estimated based on the baseline infestation category. The model selection was carried out using the Akaike Information Criterion. The comparisons of the models were carried out by estimating the likelihood-ratio test (LRT) $(p<0.05)$. Furthermore, the explanatory power of the final model was assessed by pseudoR2. The multinomial logistic model was performed using the "nnet" package.

\section{RESULTS}

\section{Data Exploration and Descriptive Analysis}

The database contains 67,909 observations corresponding to weekly samples of C. rogercresseyi abundance from 784 salmon farms from three Chilean regions (Region X [Los Lagos], Region XI [Aysén], and Region XII [Magallanes]) for 5 years (20122016). Region X presents 331 farms, Region XI maintains 397 farms, and Region XII presents 58 extant farms. The weekly number of farms varied during the study period but remained relatively stable from 2012 to 2015 (mean $=277$, sd $=30.67$ ) and declined only for 2016 ( mean $=192$, sd = 27.97) (Figure 2). The descriptive analysis and graphical tools suggest a spatial and temporal pattern in the prevalence and the growth rate (the definition of the case is at $C$. rogercresseyi abundance $>$ $3)$. The prevalence of C. rogercresseyi increased from $11.69 \%$ (95\% CI: $7.82,15.56 \%)$ at the beginning of the epidemic in February 2013 to $30.20 \%$ (95\% CI: 24.90, 35.51\%), with a peak of $42.85 \%$ (95\% CI: 36.91, 48.80\%) in April 2013. After the peak, C. rogercresseyi prevalence fell to $8.33 \%$ (95\% CI: $5.91,12.04 \%$ ) in December 2013, staying below 25\% with two peaks of $23.79 \%$ (95\% CI: 18.49, 29.08\%) in May 2014 and 16.46\% (95\% CI: 10.78, 22.13\%) in April 2016 (Figure 2). Incidence estimates considered 3,612 new cases (case per time unit [week]) during 5 years of observation, with a peak in April 2013. The growth rate before the peak is 0.001 per farm-year at risk, and that after the peak is -0.004 per farm-year at risk (Figure 3). Omitting the peak of new cases, the growth rate during these 5 years is -0.002 per farm-year at risk.

\section{Space-Time Cluster Analysis}

The multinomial space-time scan test identified 26 significant clusters $(p<0.05)$, of which approximately half $(12 / 26)$ correspond to the baseline category, whereas one is a medium infestation cluster and 13 are high infestation clusters. The number of farms per cluster varied from 4 to 39 (mean = 20 , sd $=10.72$, Figure 1 ). The most likely cluster that included farms at the baseline category had a radius of $29.44 \mathrm{~km}$, was located in Puerto Cisnes (-44.5725, -72.7172), and occurred from January to June 2013, during the peak of the occurrence pattern (Table 1, Figure 1). The medium infestation cluster was detected in the middle of the first year of study from July to December 2012, with a radius of $28.08 \mathrm{~km}$, and it was located in the northwestern portion of National Park Laguna San Rafael $(-45.7675,-74.5672)$ (Table 1, Figure 1). The high infestation cluster was detected during the peak of the occurrence pattern from January to May 2013, with a radius of $17.50 \mathrm{~km}$, and it was located in the north area of Chiloe Island $(-42.3197,-73.2691)$ (Table 1, Figure 1). The temporality and locality of the significant clusters are presented in Table 1. Most of the clusters coincide with the results of the descriptive analysis with an estimated peak during 2013.

\section{Regression Model for Associated Factors}

There was a weak correlation between continuous predictors (the relationship by Pearson correlation coefficients was $<0.7$ and not significant with a $p>0.05$ ), and numerical variables were log-transformed to access normality. The final multinomial regression model included year category, salmon species, latitude in decimal degrees, average weight, temperature, salinity, and chemotherapeutants. Table 2 shows the parameter estimators, relative risk ratios, standard error, and significance probability for the final multinomial model. Figure 4 shows the probability curves estimated by the model. Although the explicative power of the model was limited (pseudo-R2 $=12 \%$ ), the inclusion of the variables significantly improved model fitness, comparing the LRT values of the final model (LRT $=-43,566$ ) and intercept model (LRT $=-50,426$ ). Biomass, number of fish, epidemiological neighborhood, company, the number of chemical treatments (integer counts), and geographical region of Chile lacked significance within the model.

Some different risk factors are observed in medium and high $C$. rogercresseyi abundance levels, taking the baseline category as reference (including year category: 2012, species: Atlantic salmon, and chemical ingredient: azamethiphos as reference values). Diflubenzuron and cypermethrin presented no differences in risk to higher abundance levels between diflubenzuron- and azamethiphos-treated farms $(p>0.05)$, while emamectin benzoate and deltamethrin present a statistically significant risk to farms in this category $(p<0.001)$. Besides, all chemical ingredients reduce a significant risk on the medium level of infestation $(p<0.001)$. Temperature presented a slightly non-significant risk on the medium level of infestation $(p=0.08)$, while a significant risk was observed on a high level $(p<0.001)$. In the case of the year category (increase for 2013 and decrease for subsequent years), the species of fish (rainbow trout with more risk and coho salmon with lower risk), the salinity (significant 

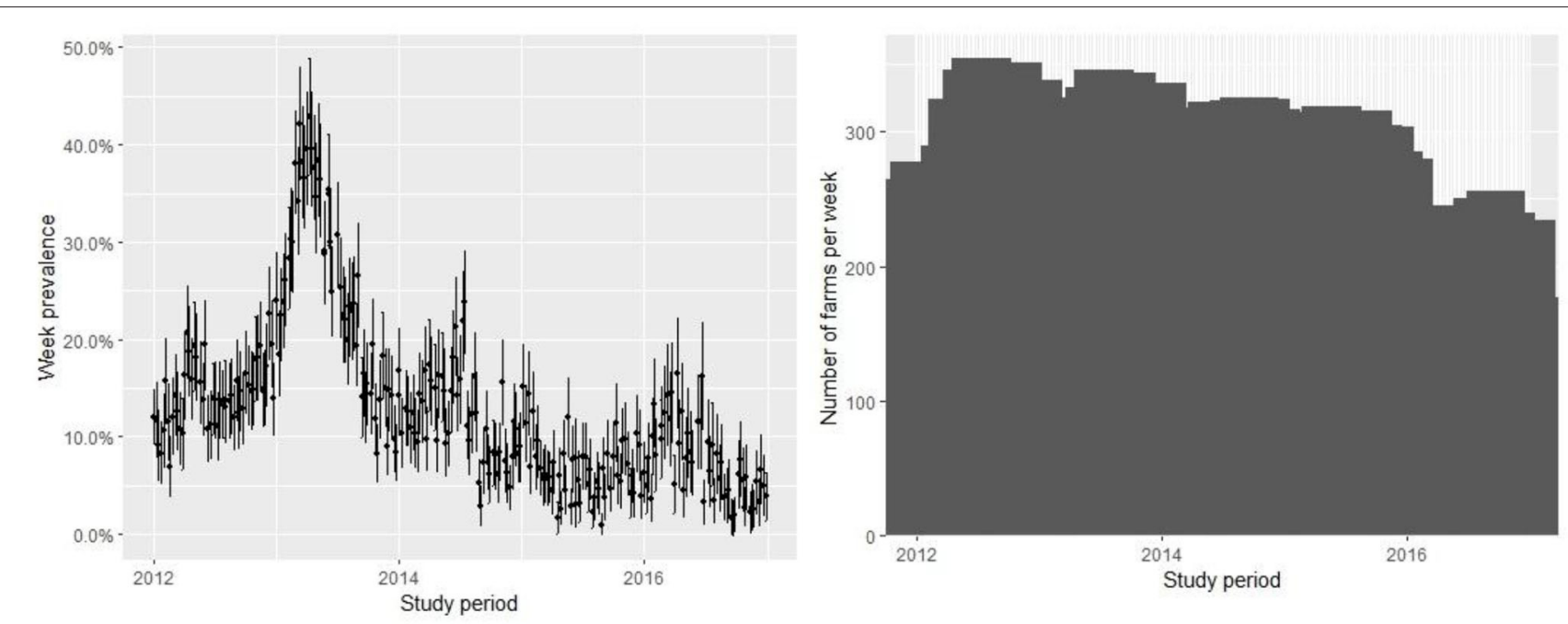

FIGURE 2 | Prevalence of farms with Caligus rogercresseyi abundance > 3 and 95\% Cl (left) and measure of active farms (right) during $2012-2016$ in southern Chile. The pattern suggests an epidemic curve of prevalence and the number of farms varied with relative stability during the study period.

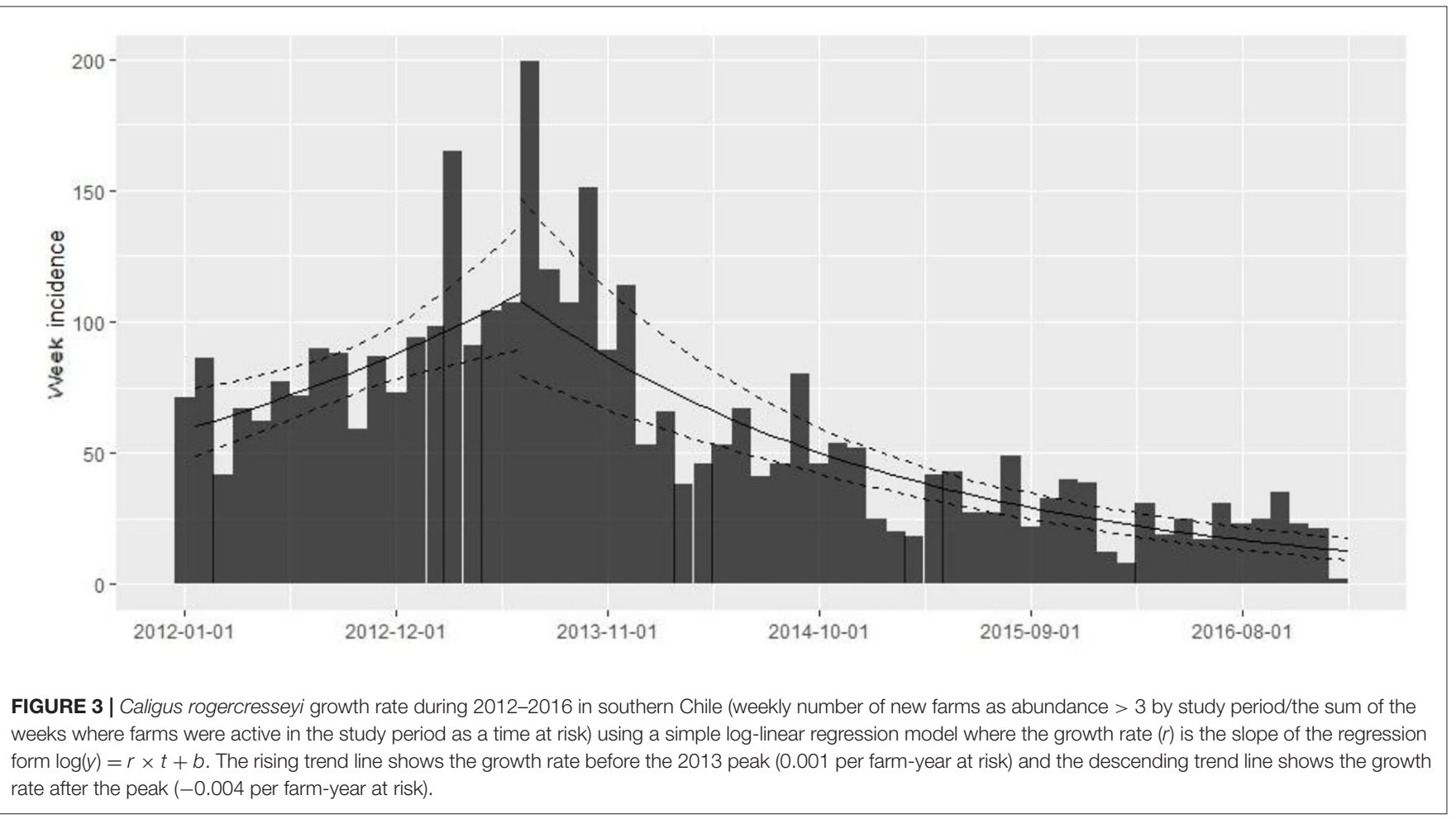

risk), the average weight (significant risk), and the latitude (lower risk), the same effect is observed in both levels of infestation.

\section{DISCUSSION}

Our results suggest that there is a $C$. rogercresseyi space-time pattern in southern Chile for the years 2012-2016. Furthermore, there are production and environmental characteristics that influence these patterns, depending on the infestation level (baseline, medium, and high) according to the multinomial regression analysis. This space-time pattern influenced by different factors shows a heterogeneous large-scale process of this parasite disease in an aquatic environment. The results from this study are consistent with the historical analysis suggesting that $C$. rogercresseyi infestations are clustered in particular areas, or even in particular areas at particular times (11). On the other hand, these findings suggest that management practices and sanitary regulations could vary and 
TABLE 1 | Clusters of Caligus rogercresseyi detected and their risk ratios from 2012 to 2016 in Region X [Los Lagos], Region XI [Aysén], and Region XII [Magallanes] of Chile.

\begin{tabular}{|c|c|c|c|c|c|c|c|c|c|c|}
\hline \multirow[b]{2}{*}{ Category } & \multirow[b]{2}{*}{ Cluster } & \multirow[b]{2}{*}{$n$} & \multicolumn{3}{|c|}{ Temporal distribution } & \multicolumn{3}{|c|}{ Spatial distribution } & \multirow[b]{2}{*}{ Risk ratio } & \multirow[b]{2}{*}{$P$} \\
\hline & & & Start & End & No. month & Latitude (S) & Longitude (W) & Radius (Km) & & \\
\hline \multirow{12}{*}{$\begin{array}{l}\text { Baseline } \\
\text { (abundance 0-2) }\end{array}$} & 3 & 41 & January 1, 2012 & June 30, 2012 & 6 & 41.6713 & 72.4222 & 28.75 & 1.99 & 0.001 \\
\hline & 4 & 39 & January 1, 2013 & June 30, 2013 & 6 & 44.5725 & 72.7172 & 29.44 & 2.03 & 0.001 \\
\hline & 7 & 29 & March 1, 2014 & August 31, 2014 & 6 & 45.4216 & 73.0222 & 28.04 & 2.03 & 0.001 \\
\hline & 9 & 20 & November 1, 2012 & April 30, 2013 & 6 & 42.3144 & 72.5119 & 19.09 & 1.98 & 0.001 \\
\hline & 12 & 15 & January 1, 2014 & June 30, 2014 & 6 & 46.1208 & 73.4502 & 26.50 & 2.02 & 0.001 \\
\hline & 15 & 12 & April 1, 2012 & August 31, 2012 & 6 & 42.5200 & 72.7075 & 19.36 & 2.02 & 0.001 \\
\hline & 20 & 12 & October 1, 2013 & March 31, 2014 & 6 & 42.9591 & 72.8102 & 29.61 & 2.02 & 0.001 \\
\hline & 22 & 13 & January 1, 2012 & June 30, 2012 & 6 & 54.1452 & 71.2166 & 28.36 & 2.02 & 0.001 \\
\hline & 23 & 3 & August 1, 2012 & January 31, 2013 & 6 & 43.7413 & 73.0097 & 2.34 & 2.01 & 0.001 \\
\hline & 24 & 12 & April 1, 2016 & September 30, 2016 & 6 & 52.6286 & 72.3722 & 28.22 & 2.01 & 0.001 \\
\hline & 25 & 17 & July 1, 2016 & December 31, 2016 & 6 & 51.9702 & 72.9463 & 24.54 & 2.01 & 0.001 \\
\hline & 26 & 4 & February 1, 2014 & July 31, 2014 & 6 & 54.4833 & 71.4780 & 26.65 & 2.01 & 0.001 \\
\hline $\begin{array}{l}\text { Medium } \\
\text { (abundance 2-4) }\end{array}$ & 19 & 20 & July 1, 2012 & December 31, 2012 & 6 & 45.7675 & 74.5672 & 28.08 & 2.15 & 0.001 \\
\hline \multirow{13}{*}{$\begin{array}{l}\text { High } \\
\text { (abundance >4) }\end{array}$} & 1 & 30 & January 1, 2013 & June 30, 2013 & 6 & 44.5980 & 74.0522 & 28.15 & 4.78 & 0.001 \\
\hline & 2 & 34 & March 1, 2013 & August 31, 2013 & 6 & 45.2186 & 73.6819 & 26.64 & 3.85 & 0.001 \\
\hline & 5 & 29 & February 1, 2013 & July 31, 2013 & 6 & 45.6766 & 73.8677 & 25.68 & 4.29 & 0.001 \\
\hline & 6 & 24 & January 1, 2013 & May 31, 2013 & 6 & 42.3197 & 73.2691 & 17.50 & 3.97 & 0.001 \\
\hline & 8 & 14 & February 1, 2013 & July 31, 2013 & 6 & 43.9661 & 73.1286 & 18.89 & 5.02 & 0.001 \\
\hline & 10 & 16 & 2015/10/1 & March 31, 2016 & 6 & 42.6355 & 73.5358 & 12.44 & 1.65 & 0.001 \\
\hline & 11 & 8 & February 1, 2013 & June 30, 2013 & 6 & 43.9119 & 73.8250 & 8.66 & 5.30 & 0.001 \\
\hline & 13 & 34 & January 1, 2013 & June 30, 2013 & 6 & 43.0636 & 73.5538 & 19.59 & 1.51 & 0.001 \\
\hline & 14 & 26 & February 1, 2013 & July 31, 2013 & 6 & 41.8163 & 73.3341 & 23.64 & 3.06 & 0.001 \\
\hline & 16 & 19 & May 1, 2012 & 2012/10/31 & 6 & 44.2472 & 73.7372 & 13.41 & 3.39 & 0.001 \\
\hline & 17 & 14 & March 1, 2013 & August 31, 2013 & 6 & 44.2113 & 74.2866 & 27.31 & 3.28 & 0.001 \\
\hline & 18 & 21 & January 1, 2013 & June 30, 2013 & 6 & 44.5641 & 73.3641 & 29.25 & 2.80 & 0.001 \\
\hline & 21 & 6 & March 1, 2013 & August 31, 2013 & 6 & 45.6566 & 73.2977 & 19.92 & 5.52 & 0.001 \\
\hline
\end{tabular}

Clusters are shown by number, and the location is depicted in Figure 1.

adapt according to the geographical location of farms across the entire area occupied by the industry. The current study also demonstrates the usefulness of space-time scan statistics (SaTScan), expressed in the identification of infestation clusters in a complex system of fjords and islands in Chile, offering a potential use as part of surveillance programs. Official notification of clusters would alert neighboring active farms to the spread of $C$. rogercresseyi, allowing them to carry out early management measures.

Space-time C. rogercresseyi variation in Chile has been reported before, but the cluster behavior from baseline farms concerning infestation had not been examined. Approximately $46 \%$ of clusters (12/26) present C. rogercresseyi abundances below the threshold of control chemical events. Some of these clusters even presented low $C$. rogercresseyi abundances during the epidemic peaks of the year 2013, as detected in the prevalence and incidence analysis (Figure 3). The estimates of the multinomial model suggest that production variable biomass, fish species, and salinity influence these baseline clusters. The density-dependent relationship between sea lice copepods and the concentration of susceptible hosts has been proposed previously (8). Our finding on the positive effect of biomass in C. rogercresseyi occurrence is consistent with those of different authors $(1,19,24,26,43,46-49)$ who suggest that it is necessary to set up a maximum level infield practice about fish density per water unit area, predisposing host susceptibility to confinement and stress increases. Bravo et al. (14) propose that a density $>10 \mathrm{~kg} / \mathrm{m}^{3}$ increases local $C$. rogercresseyi abundances; nevertheless, it is not clear whether this estimate can be applied to the growing trend of this economic activity. According to the probabilistic curves of our model (Figure 4), at more than $10,000 \mathrm{~kg}$, farms are at risk of reaching the medium level of infestation, and at $20,000 \mathrm{~kg}$ of stock density, the risk of going into the high level of infestation increases (when Atlantic salmon or rainbow trout are grown). Besides, for coho salmon, the risk increases when density reaches $40,000 \mathrm{~kg}$. In Chile, there are no regulations based on carrying capacity estimates to limit maximum fish biomass per area or water body (50). 
TABLE 2 | Results of the multinomial logistic model to quantify the association with production and environmental factors hypothesized to influence the C. rogercresseyi medium and high category in Chile during 2012-2016, compared to the baseline category as reference (year category: 2012, species: Atlantic salmon, and chemical ingredient: azamethiphos as reference values).

\begin{tabular}{|c|c|c|c|c|}
\hline $\begin{array}{l}\text { Medium } \\
\text { category } \\
\text { Abundance 2-4 }\end{array}$ & Coefficient & Std. Errors & z-stat & $P$-value \\
\hline (Intercept) & -18.100 & 0.590 & -30.680 & $<0.001$ \\
\hline 2013 & 0.192 & 0.034 & 5.667 & $<0.001$ \\
\hline 2014 & -0.516 & 0.037 & -14.007 & $<0.001$ \\
\hline 2015 & -0.395 & 0.037 & -10.677 & $<0.001$ \\
\hline 2016 & -0.428 & 0.040 & -10.703 & $<0.001$ \\
\hline $\mathrm{BE}$ & -0.400 & 0.103 & -3.897 & $<0.001$ \\
\hline CYP & -0.341 & 0.101 & -3.367 & $<0.001$ \\
\hline DEL & -0.658 & 0.092 & -7.174 & $<0.001$ \\
\hline DIL & -1.218 & 0.494 & -2.469 & 0.014 \\
\hline NA & -0.417 & 0.080 & -5.237 & $<0.001$ \\
\hline Coho & $-1.22 \mathrm{E}+01$ & 6.10E-06 & $-2.01 E+06$ & $<0.001$ \\
\hline Rainbow trout & 0.263 & 0.027 & 9.663 & $<0.001$ \\
\hline Temperature & -0.050 & 0.088 & -0.571 & 0.568 \\
\hline Salinity & 4.447 & 0.094 & 47.200 & $<0.001$ \\
\hline Average weight & 14.423 & 0.310 & 46.543 & $<0.001$ \\
\hline Latitude & -0.041 & 0.007 & -6.109 & $<0.001$ \\
\hline $\begin{array}{l}\text { High category } \\
\text { Abundance }>4\end{array}$ & Coefficient & Std. Errors & z stat & $P$-value \\
\hline (Intercept) & -30.555 & 0.899 & -33.997 & $<0.001$ \\
\hline 2013 & 0.861 & 0.048 & 17.760 & $<0.001$ \\
\hline 2014 & -0.520 & 0.058 & -9.015 & $<0.001$ \\
\hline 2015 & -0.917 & 0.063 & -14.465 & $<0.001$ \\
\hline 2016 & -0.748 & 0.065 & -11.452 & $<0.001$ \\
\hline $\mathrm{BE}$ & -0.547 & 0.136 & -4.013 & $<0.001$ \\
\hline CYP & 0.044 & 0.125 & 0.350 & 0.726 \\
\hline DEL & -0.260 & 0.113 & -2.294 & 0.022 \\
\hline DIL & -0.743 & 0.745 & -0.997 & 0.319 \\
\hline NA & -0.261 & 0.094 & -2.770 & $<0.001$ \\
\hline Coho & $-1.07 \mathrm{E}+01$ & 1.64E-05 & $-6.54 \mathrm{E}+05$ & $<0.001$ \\
\hline Rainbow trout & 0.295 & 0.041 & 7.156 & $<0.001$ \\
\hline Temperature & 1.155 & 0.130 & 8.863 & $<0.001$ \\
\hline Salinity & 5.386 & 0.154 & 34.957 & $<0.001$ \\
\hline Average weight & 21.153 & 0.441 & 47.986 & $<0.001$ \\
\hline Latitude & -0.149 & 0.009 & -16.113 & $<0.001$ \\
\hline
\end{tabular}

Coefficients, standard error, z-value, and significance probability are shown for each factor. Average weight, salinity, and temperature were log-transformed.

According to the multinomial model, rainbow trout positively influences C. rogercresseyi occurrence in baseline clusters when compared to Atlantic salmon and coho salmon. Since the beginning of the industry in Chile, a greater abundance and prevalence of $C$. rogercresseyi adult stages in rainbow trout has been observed in the field, and experiment studies compared them to Atlantic salmon $(11,13,51,52)$. However, some studies have also observed similarities in $C$. rogercresseyi susceptibility by rainbow trout and Atlantic salmon, arguing differences caused by temperature $>12^{\circ} \mathrm{C}$ and salinity $>25 \mathrm{ppt}$ (14). The susceptibility of rainbow trout to $C$. rogercresseyi could be explained by semiochemicals and mucus cell hypertrophy response (53). Besides, there is very low heritability susceptibility to $C$. rogercresseyi in rainbow trout (54). In contrast, coho salmon (as the most $C$. rogercresseyi-resistant species) has a low mucus biochemical response and a different macrophage response mechanism to $C$. rogercresseyi infestation (52).

Salinity is the environmental characteristic that reduces the occurrence of $C$. rogercresseyi in baseline clusters, giving an advantage to farms in sites with a low salinity concentration. These clusters are, spatially speaking, on the coastline, mainly in estuarine areas. This fact is consistent with the modeling of the parasitic dynamics by Mancilla-Schulz et al. (19), who compared three production sites of Region X [Los Lagos] during the years 2012-2015 and observed that the site with lower salinities presented lower $C$. rogercresseyi abundances. The tolerance of C. rogercresseyi at low salinities is according to that reported by Bravo et al. (12), who proves a sensitivity to salinities $<20$ ppt. Besides, low salinities can reduce $C$. rogercresseyi survival by up to $40 \%$. In the Northern Hemisphere, another species of sea lice (Lepeophtheirus salmonis) shows adult mortality at salinities $<12$ ppt, egg hatching problems at salinities $<15 \mathrm{ppt}$, and developmental limitations of the parasitic stages at salinities $<$ 30 ppt (55). It has been mentioned that low salinity can cause the death of the copepodid (parasitic stages) interrupting the life cycle of C. rogercresseyi (3), which explains the importance of this environmental characteristic to maintain baseline occurrence in clusters.

The medium infestation clusters (1/26) lack significant differences concerning associated factors with the baseline category. Despite medium infestation clusters not occupying estuarine areas, the spatial isolation from external sources could be the cause of this parasitic threshold (Figure 1). Therefore, biomass management and choice of more resistant salmon species could guarantee a better performance of these medium clusters for the occurrence of $C$. rogercresseyi. On the other hand, the measures implemented by the health authority involve other alternatives, such as chemical control rotation, coordination between neighboring farms to synchronize chemical control, and the inclusion of new chemical molecules for delousing. However, C. rogercresseyi populations frequently exposed to chemical molecules demonstrate changes in different molecular mechanisms associated with detoxification against pyrethroids, organophosphates, and avermectins (28). This should be taken with caution, mainly because the industry cannot dispense with chemical control, and it is rather necessary to add the intervention of fish densities within farms and the densities of neighboring farms in space-time to the available control strategies. The density-dependent dynamics of $C$. rogercresseyi represent a potent negative feedback that may limit the sanitary, economic, and environmental sustainability of salmon farming in the short term (56).

Although a number of antiparasitic treatments lack an effect, different consequences of the active ingredients in them were identified. In the baseline and medium infestation levels, all active 

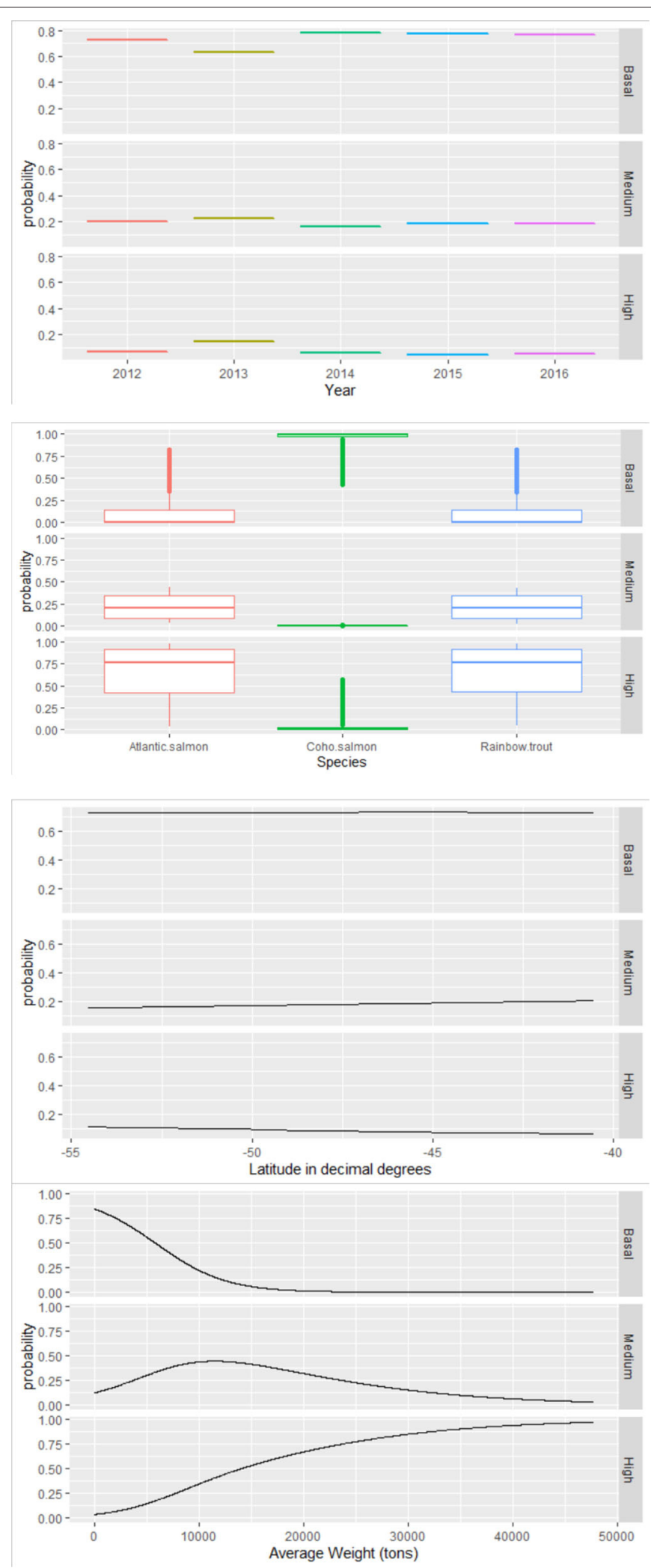

Figure 4 | Continued 

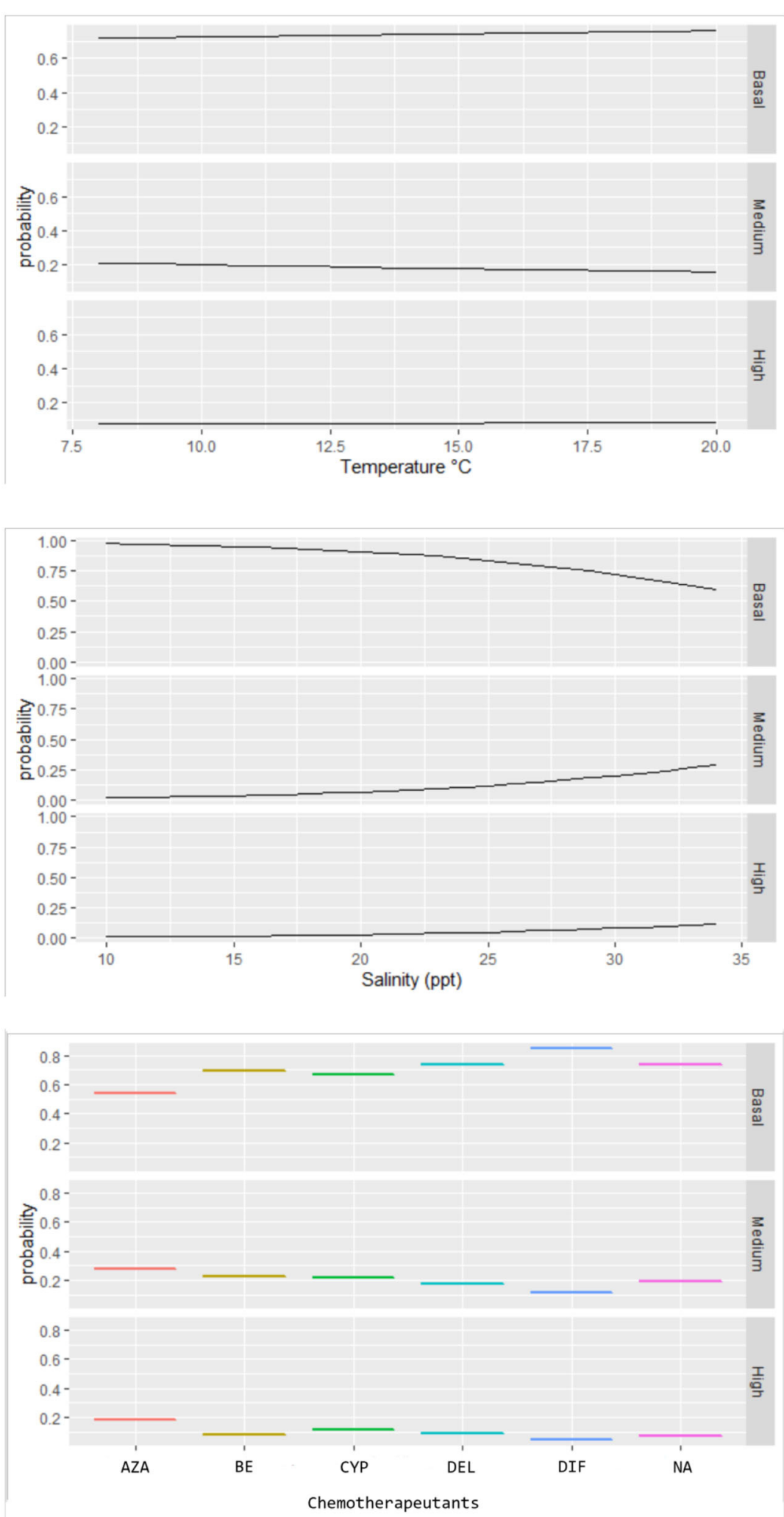

Figure 4 | Probability values estimated by the multinomial model for the effects of associated factors to infestation category of $C$. rogercresseyi (basal, medium, and high) in southern Chile during 2012-2016. The final multinomial regression model included year category, salmon species, latitude in decimal degrees, average weight, temperature, salinity, and chemotherapeutants. ${ }^{*}$ Chemotherapeutants: azamethiphos, emamectin benzoate, cypermethrin, deltamethrin, diflubenzuron, and no treatment. 
ingredients appear to have a negative effect, especially the most recent ingredient in the study period (azamethiphos). However, at the high level of $C$. rogercresseyi infestation, cypermethrin and diflubenzuron do not affect it, suggesting a loss of antiparasitic sensibility. This is consistent with those reported by different authors about the spatially structured response and resistance of pyrethroids and the broad spectrum of azamethiphos (21, $23-26,28,29)$. This makes it necessary to include spatial structure in the application of new active ingredients and even to authorize and restrict the use of active ingredients according to sensitivity bioassays in the laboratory and field studies. In addition, the use of new ingredients could be part of an exclusive application reserved for outbreaks clusters, reducing overuse and/or incorrect use. Another central aspect is to avoid unnecessary use of chemicals and respecting the time of their use according to the label (32). Currently, all authorized drugs in Chile can be applied, with rotations, in all farms that need to control the parasite.

Temperature and latitude are the factors that influence high infestation clusters (13/26). These clusters were grouped in the north of the Chiloe Island in Region X [Los Lagos] and the central zone of the inner sea of Region XI [Aysén] during 2013, coinciding with the prevalence and incidence peaks observed in this study (Figures 1, 2). The spatial pattern observed in our study is contrary to the spatial trend reported by Hamilton-West et al. (10) about how C. rogercresseyi occurrence should present a decreasing pattern according to the latitudinal gradient toward Region XI [Aysén]. In our study, we observe that high infestation clusters are mostly located in Region XI [Aysén]. Otherwise, our results are consistent with the spatiotemporal patterns observed for C. rogercresseyi resistance to chemical control. Limited effectiveness of pyrethroids against C. rogercresseyi has been reported in a retrospective study in two areas during 2012-2013, one located in northern Region $\mathrm{X}$ [Los Lagos] and the other in the central area of Region XI [Aysén] (25). However, even though we observe a heterogeneous C. rogercresseyi clustered pattern, there are unmeasured factors in the understanding of those processes behind the infestation patterns.

Temperature is the main environmental characteristic influencing high infestation clusters, since the reproductive output of C. rogercresseyi, the time of maturation for eggs, and the development of parasitic stages can be reduced in warm water (57). Besides, the life cycle of C. rogercresseyi remains stable at $4-17^{\circ} \mathrm{C}$ and is shorter in summer (January: more than 18 days) and longer in winter (July: 45 days) (3). In a comparative study between the epidemic peaks of another sea lice species, L. salmonis, in Norway and Scotland, it is postulated that an increase in temperature could increase the fitness of the parasitic stages (copepodid), explaining the increase of the infestation pressure within and between neighboring clusters (58). Another mechanism not considered in the present study, but one that could explain infestation pressure, is the higher current circulation in Region XI [Aysén] channels, transporting the parasitic stages within high infestation clusters (59). The use of deeper cages is a possible measure in these clusters, avoiding surface temperature and the parasitic stage, which is generally found closer to the surface of the water. However, the influence of temperature in high infestation clusters is a cause for concern, due to the climate, changing predictions like the increase of solar radiation reaching the ocean waters, and air temperature near the ocean surface (2).

The descriptive results show that there has been a notable change in C. rogercresseyi prevalence and incidence over the 5 years studied. A possible explanation may be the loss of sensitivity to chemotherapy, causing an increase in the number of cases during 2013. Previously, it has been suggested that efficacy was lower for pyrethroids (deltamethrin and cypermethrin) using $C$. rogercresseyi females collected from Puerto Montt during July of 2012 (60). Besides, azamethiphos was approved as a new antiparasitic option in May 2013, explaining the decrease in cases for the years 2014-2016 (27). Furthermore, a bioassay to monitor the sensitivity of $C$. rogercresseyi males and females to deltamethrin, cypermethrin, and azamethiphos during 20132014 suggests a spatial variability response to deltamethrin and cypermethrin due to the continuous treatments applied on farms (29).

The findings of this retrospective study suggest the importance of addressing the density-dependent aspect as a possible IPM tool in the C. rogercresseyi clustering infestation process. The number of susceptible individuals per unit area is a cornerstone of the transmission in infectious disease dynamics. The open system waters in the ocean salmonid production facilities present a connection with wild fish population carrying the parasite. In Chile, C. rogercresseyi was transmitted to farmed fish by the native Rock cod (Eleginops maclovinus) (61) and Chilean silverside (Odontesthes regia) (62). Stocking density thresholds can decrease host stress and the impact of parasitic copepods (5). Moreover, there is a spatial density function based on the distance between active farms. Sanitary and policy management should promote attention to empirical evidence about host density threshold experiments and how this evidence is connected to endemic and epidemic C. rogercresseyi patterns.

\section{DATA AVAILABILITY STATEMENT}

The raw data supporting the conclusions of this article will be made available by the authors, without undue reservation.

\section{AUTHOR CONTRIBUTIONS}

ML-L wrote the manuscript and analyzed and interpreted data. JE-D and NZ-V collected data and wrote the manuscript. DR wrote the manuscript and prepared the tables and figures. JA wrote the manuscript and interpreted data analysis. FM wrote the manuscript, conceived and planned the study, and acquired funds. All authors contributed to the article and approved the submitted version.

\section{FUNDING}

This work was supported by the EPIVET project 201707240090, which was financed by SalmonChile and Sernapesca 
through the Fondo Inversion Estrategica (FIE-2015V014) from the Ministry of Economy. Partial fund was also obtained by the National Fund for Scientific and Technological Development of the Government of Chile (FONDECYT), through the grant: FONDECYT regular No1191675.

\section{REFERENCES}

1. Dresdner J, Chávez C, Quiroga M, Jiménez D, Artacho P, Tello A. Impact of Caligus treatments on unit costs of heterogeneous salmon farms in Chile. Aquac Econ Manag. (2019) 23:1-27. doi: 10.1080/13657305.2018.1449271

2. Soto D, León-Muñoz J, Dresdner J, Luengo C, Tapia FJ, Garreaud R. Salmon farming vulnerability to climate change in southern Chile: understanding the biophysical, socioeconomic and governance links. Rev Aquac. (2019) 11:354-74. doi: 10.1111/raq.12336

3. González L, Carvajal J. Life cycle of Caligus rogercresseyi, (Copepoda: Caligidae) parasite of Chilean reared salmonids. Aquaculture. (2003) 220:10117. doi: 10.1016/S0044-8486(02)00512-4

4. Gallardo-Escárate C, Valenzuela-Muñoz V, Nuñez-Acuña G, ValenzuelaMiranda D, Gonçalves AT, Escobar-Sepulveda H, et al. Chromosome-scale genome assembly of the sea louse Caligus rogercresseyi by SMRT sequencing and Hi-C analysis. Sci Data. (2021) 8:1-12. doi: 10.1038/s41597-021-00842-w

5. Johnson SC, Treasurer JW, Bravo S, Nagasawa K, Kabata Z. A review of the impact of parasitic copepods on marine aquaculture. Zool Stud. (2004) 43:229-43. Available online at: http://zoolstud.sinica.edu.tw/Journals/ 43.2/229.pdf

6. González Gómez MP, Marín Arribas SL, Vargas-Chacoff L. Stress response of Salmo salar (Linnaeus 1758) facing low abundance infestation of Caligus rogercresseyi (Boxshall \& Bravo 2000), an object in the tank, and handling. $J$ Fish Dis. (2016) 39:853-65. doi: 10.1111/jfd.12419

7. González MP, Muñoz JLP, Valerio V, Vargas-Chacoff L. Effects of the ectoparasite Caligus rogercresseyi on Salmo salar blood parameters under farm conditions. Aquaculture. (2016) 457:2934. doi: 10.1016/j.aquaculture.2016.01.027

8. Costello MJ. Ecology of sea lice parasitic on farmed and wild fish. Trends Parasitol. (2006) 22:475-83. doi: 10.1016/j.pt.2006.08.006

9. Rozas M, Asencio G. Assessment of epidemiologic situation of caligiasis in chile: toward to effective control strategy. Salmociencia. (2007) 2:43-59.

10. Hamilton-West C, Arriagada G, Yatabe T, Valdés P, Hervé-Claude LP, Urcelay S. Epidemiological description of the sea lice (Caligus rogercresseyi) situation in southern Chile in August 2007. Prev Vet Med. (2012) 104:3415. doi: 10.1016/j.prevetmed.2011.12.002

11. Zagmutt-Vergara FJ, Carpenter TE, Farver TB, Hedrick RP. Spatial and temporal variations in sea lice (Copepoda: Caligidae) infestations of three salmonid species famed in net pens in southern Chile. Dis Aquat Organ. (2005) 64:163-73. doi: 10.3354/dao064163

12. Bravo S, Pozo V, Silva MT. The tolerance of Caligus rogercresseyi to salinity reduced in southern Chile. Bull Eur Assoc Fish Pathol. (2008) 28:198-206. Available online at: https://eafp.org/download/2008-Volume28/Issue\%205/ 28_198.pdf

13. Yatabe T, Arriagada G, Hamilton-West C, Urcelay S. Risk factor analysis for sea lice, Caligus rogercresseyi, levels in farmed salmonids in southern Chile. $J$ Fish Dis. (2011) 34:345-54. doi: 10.1111/j.1365-2761.2011.01238.x

14. Bravo S, Silva MT, Treasurer J. Factors affecting the abundance of Caligus rogercresseyi (Boxshall and Bravo) on farmed salmonids in Chile in the period 2006-2007. Aquaculture. (2014) 434:456-61. doi: 10.1016/j.aquaculture.2014.09.009

15. Zalcman E, Burroughs A, Meyer A, Hillman A, Sadler R, Madin B, et al. Sea lice infestation of salmonids in Chile between 2011 and 2017: use of regulatory data to describe characteristics and identify risk factors. Aquaculture. (2021) 530:735752. doi: 10.1016/j.aquaculture.2020.735752

16. Chávez C, Dresdner J, Figueroa Y, Quiroga M. Main issues and challenges for sustainable development of salmon farming in Chile: a socio-economic perspective. Rev Aquac. (2019) 11:403-421. doi: 10.1111/raq.12338

\section{ACKNOWLEDGMENTS}

The authors thank Salmon Technological Institute and the authority Sernapesca for access to information from the Specific Sanitary Program for the Surveillance and Control of Caligidosis (SSPSC Caligidosis).

17. Asche F, Hansen H, Tveteras R, Tveteras S. The salmon disease crisis in Chile. Mar Resour Econ. (2009) 24:405-11. doi: 10.1086/mre.24.4.42629664

18. Anderson RM, May RM. Infectious Diseases of Humans: Dynamics and Control. New York, NY: Oxford University Press (1991).

19. Mancilla-Schulz J, Marín SL, Molinet C. Dynamics of Caligus rogercresseyi (Boxshall \& Bravo, 2000) in farmed Atlantic salmon (Salmo salar) in southern Chile: are we controlling sea lice? J Fish Dis. (2019) 42:35769. doi: 10.1111/jfd.12931

20. González MT, Leiva NV, Sepúlveda F, Asencio G, Baeza JA. Genetic homogeneity coupled with morphometric variability suggests high phenotypic plasticity in the sea louse Caligus rogercresseyi (Boxshall and Bravo, 2000), infecting farmed salmon (Salmo salar) along a wide latitudinal range in southern Chile. J Fish Dis. (2021) 44:633-8. doi: 10.1111/jfd.13341

21. Bravo S, Sevatdal S, Horsberg TE. Sensitivity assessment in the progeny of Caligus rogercresseyi to emamectin benzoate. Bull Eur Assoc Fish Pathol. (2010) 30:99-105. Available online at: http://eafp.org/download/2010Volume30/Issue\%203/Bravo.pdf

22. Sernapesca. Productos antiparasitarios para el control de caligidosis en salmonideos con registro del servicio agrícola y ganadero (sag). (2019) 1. Available online at: http://www.sernapesca.cl/sites/default/files/productos_ antiparasitarios_para_el_control_de_caligidosis_en_salmonideos_con_ registro_del_sag.pdf

23. Arriagada GA, Stryhn H, Campistó JL, Rees EE, Sanchez J, Ibarra R, et al. Evaluation of the performance of pyrethroids over different life stages of C. rogercresseyi in southern Chile. Aquaculture. (2014) 426-427:2317. doi: 10.1016/j.aquaculture.2014.02.007

24. Arriagada GA, Marín SL. Evaluating the spatial range of the effect of synchronized antiparasitic treatments on the abundance of sea lice Caligus rogercresseyi (Boxshall \& Bravo, 2000) in Chile. Aquac Res. (2018) 49:81631. doi: 10.1111/are.13513

25. Arriagada G, Sanchez J, Stryhn H, Vanderstichel R, Campistó JL, Ibarra $\mathrm{R}$, et al. A multivariable assessment of the spatio-temporal distribution of pyrethroids performance on the sea lice Caligus rogercresseyi in Chile. Spat Spatiotemporal Epidemiol. (2018) 26:1-13. doi: 10.1016/j.sste.2018.04.004

26. Bravo S, Treasurer J, Sepulveda M, Lagos C. Effectiveness of hydrogen peroxide in the control of Caligus rogercresseyi in Chile and implications for sea louse management. Aquaculture. (2010) 303:22-7. doi: 10.1016/j.aquaculture.2010.03.007

27. Jimenez DF, Ibarra R, Artacho P, Primus AE, Tello A. Prolonging Azamethiphos bath increases the effectiveness of field treatments against Caligus rogercresseyi in Atlantic salmon in Chile (Salmo salar). Aquaculture. (2018) 493:186-91. doi: 10.1016/j.aquaculture.2018.04.034

28. Gallardo-Escárate C, Arriagada G, Carrera C, Gonçalves AT, Nuñez-Acuña G, Valenzuela-Miranda D, et al. The race between host and sea lice in the Chilean salmon farming: a genomic approach. Rev Aquac. 11:32539. doi: 10.1111/raq. 12334

29. Marín SL, Ibarra R, Medina MH, Jansen PA. Sensitivity of Caligus rogercresseyi (Boxshall and Bravo 2000) to pyrethroids and azamethiphos measured using bioassay tests-A large scale spatial study. Prev Vet Med. (2015) 122:33-41. doi: 10.1016/j.prevetmed.2015.09.017

30. Marín SL, Sepúlveda F, Carvajal J, George-Nascimento M. The feasibility of using Udonella sp. (Platyhelminthes: Udonellidae) as a biological control for the sea louse Caligus rogercresseyi, Boxshall and Bravo 2000, (Copepoda: Caligidae) in southern Chile. Aquaculture. (2002) 208:11-21. doi: 10.1016/S0044-8486(01) 00715-3

31. Pullan RL, Sturrock HJW, Soares Magalhães RJ, Clements ACA, Brooker SJ. Spatial parasite ecology and epidemiology: 
a review of methods and applications. Parasitology. 139:1870-87. doi: 10.1017/S0031182012000698

32. Brooks KM. Considerations in developing an integrated pest management programme for control of sea lice on farmed salmon in Pacific Canada. J Fish Dis. (2009) 32:59-73. doi: 10.1111/j.1365-2761.2008.01013.x

33. Jackson D, Moberg O, Stenevik Djupevåg EM, Kane F, Hareide H. The drivers of sea lice management policies and how best to integrate them into a risk management strategy: an ecosystem approach to sea lice management. J Fish Dis. (2018) 41:927-33. doi: 10.1111/jfd.12705

34. Montory JA, Cumillaf JP, Gebauer P, Urbina M, Cubillos VM, Navarro $\mathrm{JM}$, et al. Early development and metabolic rate of the sea louse Caligus rogercresseyi under different scenarios of temperature and pCO2. Mar Environ Res. (2020) 162:105154. doi: 10.1016/j.marenvres.2020.105154

35. Boxaspen K. A review of the biology and genetics of sea lice. ICES J Mar Sci. (2006) 63:1304-16. doi: 10.1016/j.icesjms.2006.04.017

36. Pfeiffer DU, Robinson TP, Stevenson M, Stevens KB, Rogers DJ, Clemens ACA. Spatial Analysis in Epidemiology. Oxford: Oxford University Press (2008).

37. Sernapesca. Caligus Surveillance Program for Chile. (2015) Available online at: http://www.sernapesca.cl/sites/default/files/res_013_2015.pdf

38. Sernapesca. Caligus Surveillance Guide for Chile. (2015) Available online at: http://www.sernapesca.cl/sites/default/files/res_013_2015.pdf

39. Bravo S, Acuicultura I De, Austral U, Montt P. Notes Sea lice in Chilean salmon farms. Health Welfare. (2003) 23:197-200. Available online at: http:// eafp.org/download/2003-Volume23/Issue\%204/23_197.pdf

40. R Developmen Core Team. $R$ : A Language and Environment for Statistical Computing. Vienna: R Foundation for Statistical Computing (2021). Available online at: www.R-project.org/

41. Kamvar ZN, Cai J, Pulliam JRC, Schumacher J, Jombart T. Open Peer Review Epidemic curves made easy using the $\mathrm{R}$ package incidence [version 1; peer review: 1 approved, 2 approved with reservations]. F1000Res. (2019) 8:139. doi: 10.12688/f1000research.18002.1

42. Jung I, Kulldorff M, Richardc OJ. A spatial scan statistic for multinomial data Inkyung. Stat Med. (2010) 29:1910-8. doi: 10.1002/sim.3951

43. Kristoffersen AB, Rees EE, Stryhn H, Ibarra R, Campisto JL, Revie CW, et al. Understanding sources of sea lice for salmon farms in Chile. Prev Vet Med. (2013) 111:165-75. doi: 10.1016/j.prevetmed.2013.03.015

44. Hilbe JM. Logistic Regression Models. Boca Raton, FL: CRC Press (2009). doi: 10.1201/9781420075779

45. Zuur AF, Ieno EN, Elphick CS. A protocol for data exploration to avoid common statistical problems. Methods Ecol Evol. (2010) 1:314. doi: 10.1111/j.2041-210X.2009.00001.x

46. Molinet C, Cáceres M, Teresa M, Carvajal J, Asencio G, Díaz M, et al. Population dynamic of early stages of Caligus rogercresseyi in an embayment used for intensive salmon farms in Chilean inland seas. Aquaculture. (2011) 312:62-71. doi: 10.1016/j.aquaculture.2010.12.010

47. Bravo S, Nuñez M, Silva MT. Efficacy of the treatments used for the control of Caligus rogercresseyi infecting Atlantic salmon, Salmo salar L., in a new fish-farming location in Region XI, Chile. J Fish Dis. (2013) 36:2218. doi: $10.1111 /$ jfd. 12023

48. Helgesen KO, Bravo S, Sevatdal S, Mendoza J, Horsberg TE. Deltamethrin resistance in the sea louse Caligus rogercresseyi (Boxhall and Bravo) in Chile: bioassay results and usage data for antiparasitic agents with references to Norwegian conditions. J Fish Dis. (2014) 37:877-90. doi: 10.1111/jfd.12223

49. Soto D, Norambuena F. Evaluation of salmon farming effects on marine systems in the inner seas of southern Chile: a large-scale mensurative experiment. J Appl Ichthyol. (2004) 20:493-501. doi: 10.1111/j.1439-0426.2004.00602.x

50. Fuentes M, Montes RM, Soto D, Le J. Environmental issues in Chilean salmon farming : a review. (2019) 375-402. doi: 10.1111/raq.12337

51. González L, Carvajal J, George-Nascimento M. Differential infectivity of Caligus flexispina (Copepoda, Caligidae) in three farmed salmonids in Chile. Aquaculture. (2000) 183:13-23. doi: 10.1016/S0044-8486(99)00282-3
52. Valenzuela-Muñoz V, Boltaña S, Gallardo-Escárate C. Comparative immunity of Salmo salar and Oncorhynchus kisutch during infestation with the sea louse Caligus rogercresseyi: an enrichment transcriptome analysis. Fish Shellfish Immunol. (2016) 59:276-87. doi: 10.1016/j.fsi.2016.10.046

53. Fast MD, Ross NW, Mustafa A, Sims DE, Johnson SC, Conboy GA, et al. Susceptibility of rainbow trout Oncorhynchus mykiss, Atlantic salmon Salmo salar and coho salmon Oncorhynchus kisutch to experimental infection with sea lice Lepeophtheirus salmonis. Dis Aquat Organ. (2002) 52:5768. doi: 10.3354/dao052057

54. Bassini LN, Lhorente JP, Oyarzún $M$, Bangera R, Yáñez JM, Neira R. Genetic parameters for Piscirickettsia salmonis resistance, sea lice (Caligus rogercresseyi) susceptibility and harvest weight in rainbow trout (Oncorhynchus mykiss). Aquaculture. (2019) 510:276-82. doi: 10.1016/j.aquaculture.2019.05.008

55. Brooks KM. The effects of water temperature, salinity, and currents on the survival and distribution of the infective copepodid stage of sea lice (Lepeophtheirus salmonis) originating on atlantic salmon farms in the Broughton Archipelago of British Columbia, Canada. Rev Fish Sci. (2005) 13:177-204. doi: 10.1080/10641260500207109

56. Jansen PA, Kristoffersen AB, Viljugrein H, Jimenez D, Aldrin M, Stien A. Sea lice as a density-dependent constraint to salmonid farming. Proc R Soc B Biol Sci. (2012) 279:2330-8. doi: 10.1098/rspb.2012.0084

57. Bravo S. The reproductive output of sea lice Caligus rogercresseyi under controlled conditions. Exp Parasitol. (2010) 125:514. doi: 10.1016/j.exppara.2009.12.001

58. Heuch PA, Revie CW, Gettinby G. A comparison of epidemiological patterns of salmon lice, Lepeophtheirus salmonis, infections on farmed Atlantic salmon, Salmo salar L., in Norway and Scotland. J Fish Dis. (2003) 26:53951. doi: 10.1046/j.1365-2761.2003.00490.x

59. Niklitschek EJ, Soto D, Lafon A, Molinet C, Toledo P. Southward expansion of the Chilean salmon industry in the Patagonian Fjords: main environmental challenges. Rev Aquac. (2013) 5:172-95. doi: 10.1111/raq.12012

60. Marín SL, Martin R, Lewis R. Effects of Caligus rogercresseyi (Boxshall \& Bravo 2000) chalimus stage condition (dead, moribund, live) on the estimates of Cypermethrin BETAMAX® efficacy. Aquac Res. (2015) 46:306. doi: 10.1111/are. 12460

61. Bravo S, Erranz F, Silva MT. Comparison under controlled conditions of the life cycle of Lepeophtheirus mugiloidis and Caligus rogercresseyi, parasites of the Patagonian blenny Eleginops maclovinus. Aquac Res. (2021) 17. doi: $10.1111 /$ are. 15258

62. Carvajal J, González L, George-Nascimento M. Native sea lice (Copepoda: Caligidae) infestation of salmonids reared in netpen systems in southern Chile. Aquaculture. (1998) 166:241-6. doi: 10.1016/S0044-8486(98)00301-9

Conflict of Interest: The authors declare that the research was conducted in the absence of any commercial or financial relationships that could be construed as a potential conflict of interest.

Publisher's Note: All claims expressed in this article are solely those of the authors and do not necessarily represent those of their affiliated organizations, or those of the publisher, the editors and the reviewers. Any product that may be evaluated in this article, or claim that may be made by its manufacturer, is not guaranteed or endorsed by the publisher.

Copyright $\odot 2021$ Lepe-López, Escobar-Dodero, Rubio, Alvarez, Zimin-Veselkoff and Mardones. This is an open-access article distributed under the terms of the Creative Commons Attribution License (CC BY). The use, distribution or reproduction in other forums is permitted, provided the original author(s) and the copyright owner(s) are credited and that the original publication in this journal is cited, in accordance with accepted academic practice. No use, distribution or reproduction is permitted which does not comply with these terms. 